\title{
F-theory from fundamental five-branes
}

\author{
William D. Linch III ${ }^{a}$ and Warren Siegel ${ }^{b}$ \\ ${ }^{a}$ Center for String and Particle Theory, Department of Physics, \\ University of Maryland at College Park, \\ College Park, MD 20742-4111, U.S.A. \\ ${ }^{b}$ C.N. Yang Institute for Theoretical Physics, State University of New York, \\ Stony Brook, NY 11794-3840, U.S.A. \\ E-mail: wdlinch3@gmail.com, siegel@insti.physics.sunysb.edu
}

ABSTRACT: We describe the worldvolume for the bosonic sector of the lower-dimensional Ftheory that embeds $4 \mathrm{D}, \mathrm{N}=1 \mathrm{M}$-theory and the $3 \mathrm{D}$ Type II superstring. The worldvolume (5-brane) theory is that of a single $6 \mathrm{D}$ gauge 2-form $X_{M N}\left(\sigma^{P}\right)$ whose field strength is selfdual. Thus unlike string theory, the spacetime indices are tied to the worldsheet ones: in the Hamiltonian formalism, the spacetime coordinates are a $\mathbf{1 0}$ of the GL(5) of the $5 \sigma$ 's (neglecting $\tau$ ). The current algebra gives a rederivation of the F-bracket. The backgroundindependent subalgebra of the Virasoro algebra gives the usual section condition, while a new type of section condition follows from Gauß's law, tying the worldvolume to spacetime: solving just the old condition yields M-theory, while solving only the new one gives the manifestly T-dual version of the string, and the combination produces the usual string. We also find a covariant form of the condition that dimensionally reduces the string coordinates.

KEYwords: F-Theory, M-Theory, p-branes, String Duality

ARXiv EPrint: 1502.00510 


\section{Contents}

$\begin{array}{llr}1 & \text { Introduction } & 1\end{array}$

2 Currents and constraints 2

$\begin{array}{lll}3 & \text { Algebras and gauge symmetries } & 4\end{array}$

$\begin{array}{lll}4 & \text { Backgrounds } & 6\end{array}$

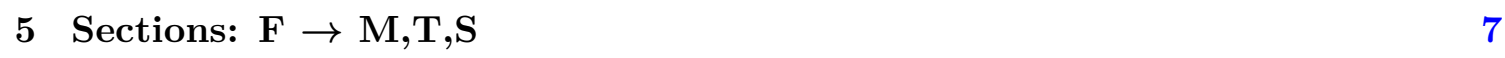

$\begin{array}{lll}6 & \text { Conclusions } & 7\end{array}$

\section{Introduction}

We continue our considerations $[1,2]$ of how string theory (S-theory) relates to its manifestly T-dual formulation (T-theory), M-theory, and the interpretation of F-theory as the manifestly STU-dual version of all these. These extensions of string theory all give rise to additional target space coordinates. For M-theory, this the familiar eleventh dimension arising in the strong-coupling limit of IIA [3] and heterotic $\mathrm{E}_{8}$ strings [4]. For manifestly T-duality-invariant theories they are the coordinates of double field theory for the winding modes of compactified strings [5-7]. (Generalized Geometry is a further simplification of this in which the winding coordinates are truncated.)

In its original form, similar coordinates were conjectured for F-theory, as a review of section 3 of the foundational reference [8] shows. Indeed, even the string worldvolume was conjectured to be extended to fundamental brane with $2+2$ signature. (The modern use of the term often refers to type IIB backgrounds that are referred to in [8] as "evidence" for the existence of such a spacetime- and worldvolume-extended theory.) In this paper, we will continue to apply the term as originally intended (and extend it to lower-dimensional "non-critical" versions of that idea). In particular, the models will be of F-theory type if the U-duality group is realized on the algebra of world-volume currents.

So far all the work on this F-theory [1, 2, 9-19] has described only the massless sector. However, T-theory was originally derived from string current algebra [5-7]. In this paper we approach F-theory from its formulation as a fundamental 5-brane, using current algebra to derive its symmetries, and how they act on a massless background. This fundamental 5-brane is a dynamical one, meant to be first-quantized (and maybe second) in a manner similar to the string. (Details of the dynamics will be left for the future.) In particular, in addition to the target space coordinates and their exceptional extensions, the spectrum will contain infinitely many massive modes. 
Our treatment of fundamental branes also differs from previous versions [20-26] in that the worldvolume fields $X_{M N}\left(\sigma^{P}\right)$ describing spacetime coordinates are gauge fields. Also, the currents are all linear in $X$, unlike treatments based on consideration of Wess-Zumino terms [14-16]. For the case of F-theory embedding the 3D string, this gauge field is the $6 \mathrm{D}$ gauge 2-form with selfdual field strength. The worldvolume indices on this gauge field $X$ (actually the 5D indices remaining in a temporal gauge) thus become identified with spacetime indices. Analysis of the current algebra of this theory naturally leads to the spacetime gauge fields of the massless sector of F-theory, their gauge transformations, the F-bracket resulting from their algebra, the F-section condition, etc. Just as the indices of the gauge field tie the worldvolume to spacetime, so does the 2-form's Gauß law, which adds a constraint to the generalized Virasoro algebra as well as a new section condition that reduces $\sigma$ as well as $x$.

\section{Currents and constraints}

Covariant selfdual 6D field theory has been described previously in terms of an action [27]. For simplicity, we start here in a "conformal gauge" where both the 6D metric and Lagrange multiplier for selfduality have been fixed, using the action only to define the Hamiltonian formalism, which we find convenient for our analysis.

The action is then, in Lagrangian form,

$$
S=\frac{1}{12} \int F_{M N P} F^{M N P} d^{6} \sigma
$$

with $F_{M N P}=\frac{1}{2} \partial_{[P} X_{M N]}$ and $F_{M N P}^{( \pm)}=F_{M N P} \pm \frac{1}{3 !} \epsilon_{M N P}{ }^{Q R S} F_{Q R S}$. The momentum conjugate to $X^{m n}$ is $P_{m n}$. In Hamiltonian form $\left(\sigma^{M} \rightarrow \tau, \sigma^{m}\right)$

$$
\begin{aligned}
S & =-\int \frac{1}{2} P_{m n} \partial_{\tau} X^{m n} d^{5} \sigma d \tau+\int H d \tau \\
H & =\int\left(\frac{1}{4} P_{m n} P^{m n}+\frac{1}{12} F_{m n p} F^{m n p}+X^{\tau m} \partial^{n} P_{m n}\right) d^{5} \sigma,
\end{aligned}
$$

where the field strengths in Hamiltonian language are

$$
F_{m n p}=\frac{1}{2} \partial_{[p} X_{m n]} \quad \text { and } \quad P_{m n}=F_{\tau m n} .
$$

The selfdual field strengths are the currents for the covariant derivatives [28]

$$
\triangleright_{m n}:=F_{\tau m n}^{(+)}=P_{m n}+\frac{1}{2} \epsilon_{m n p q r} \partial^{r} X^{p q},
$$

while the antiselfdual field strengths are the symmetry currents

$$
\tilde{\triangleright}_{m n}:=F_{\tau m n}^{(-)}=P_{m n}-\frac{1}{2} \epsilon_{m n p q r} \partial^{r} X^{p q} .
$$

As usual (cf. electromagnetism) the time components of the gauge field $X$ become Lagrange multipliers. After using them to identify the constraint (Gauß's law), we eliminate 
them by choosing a temporal gauge. The Virasoro algebra is then defined by the energymomentum tensor for the selfdual field strength

$$
\mathcal{T}_{M N}=\frac{1}{4} F_{M}{ }^{P Q} F_{N P Q} \quad \Rightarrow \quad \mathcal{T}_{M N}^{(+)}=\frac{1}{8} F_{M}^{(+)} P Q F_{N P Q}^{(+)} .
$$

(The unusual normalization is consequence of our definition of $F_{M N P}^{(+)}$) This is symmetric and traceless. Its Hamiltonian components $\left(\mathcal{T}^{(+) m n}, \mathcal{T}^{(+) \tau m}, \mathcal{T}^{(+) \tau \tau}\right)$ are

$$
\mathcal{T}_{m n}=\eta_{m n} \mathcal{T}-\frac{1}{2} \eta^{p q} \triangleright_{m p} \triangleright_{n q}, \quad \mathcal{S}^{r}=\frac{1}{16} \epsilon^{r m n p q} \triangleright_{m n} \triangleright_{p q}, \quad \mathcal{T}=\frac{1}{8} \eta^{m p} \eta^{n q} \triangleright_{m n} \triangleright_{p q} .
$$

The Gauß constraint is

$$
\mathcal{U}_{m}:=\partial^{n} P_{m n}
$$

For purposes of analyzing kinematics, we need consider only those constraints that are $\mathrm{GL}(5)$ covariant, that is, need not involve the $\mathrm{SO}(3,2)$ metric $\eta_{m n}$. (This requires treating contravariant indices on $X^{m n}$ as opposite to those on $\sigma_{m}$.) This is the subset that's background independent, since the background is introduced as a $\mathrm{GL}(5) / \mathrm{SO}(3,2) \mathrm{GL}(1)$ element to break GL(5) to $\mathrm{SO}(3,2)$. Various section-like conditions can then be introduced by replacing some string coordinates in these constraints with their zero-modes [29]:

$$
\begin{array}{rlrl}
\text { Virasoro } & \mathcal{S}^{m} & =\frac{1}{16} \epsilon^{m n p q r} \triangleright_{n p} \triangleright_{q r} & \\
\text { dimensional reduction } & \stackrel{\circ}{\mathcal{S}^{m}}:=\frac{1}{4} \epsilon^{m n p q r} p_{n p} P_{q r} & \mathcal{U}_{m}=\partial^{n} P_{m n} \\
\text { section condition } & \mathcal{S}_{\circ}^{m} & :=\frac{1}{8} \epsilon^{m n p q r} p_{n p} p_{q r} & \mathcal{U}_{\circ}:=\partial^{n} p_{m n}
\end{array}
$$

where the section conditions are to be interpreted as being applied as both

$$
A B=0 \quad \Rightarrow \quad A B f=(A f)(B g)=0
$$

for $f, g$ that are functions of $\sigma$ or $X(\sigma)$ (for hitting with $\partial^{m}$ ) or functions of $x$ or $X(\sigma)$ (for hitting with $\left.p_{m n}\right)$.

We thus have 3 types of conditions:

1. We generalized (the background independent part of) the string Virasoro algebra with the generators $\mathcal{S}^{m}$ of coordinate transformations for the $5 \sigma$ 's.

2. We treat Gauß's law $\mathfrak{U}_{m}$, which arises because the $6 \mathrm{D} X$ is a gauge field, as a dimensional reduction condition since it's linear in the string variables. We also have a new covariant dimensional reduction condition $\stackrel{\circ}{\mathcal{S}}^{m}$. (It simplified using $\mathcal{U}_{\mathrm{o}}$. Since $\partial^{[m} \partial^{n]}=0$, both dimensional reduction conditions can be written with $P$ replaced with either $\triangleright$ or $\tilde{\nabla}$ : the latter allows them to commute with Virasoro.) It replaces $P_{L}-P_{R}$ used in the manifestly T-dual version of the string that has doubled coordinates. (This reduces to $\eta^{m n} p_{m} P_{n}$ in that formalism. Dimensional reduction for doubled coordinates was invented in [30]. T-theory with doubled selfdual scalars was considered in [31]. T-theory with both selfdual and anti-selfdual scalars was attempted in $[32,33]$. 
3. The section conditions include $\mathcal{S}$, originally found by closure of gauge transformations (see below) in F-gravity [9], and a new one $\mathcal{U}$ that mutually restricts $x$ and $\sigma$. (The former condition reduces to the original section condition $\frac{1}{2} \eta^{m n} p_{m} p_{n}$ in T-theory [57].)

\section{Algebras and gauge symmetries}

Using the Poisson bracket

$$
\left[P_{m n}(1), X^{p q}(2)\right]=-i \delta_{[m}^{p} \delta_{n]}^{q} \delta(1-2)
$$

we find the algebra

$$
\begin{aligned}
& {\left[\triangleright_{m n}(1), \triangleright_{p q}(2)\right]=2 i \epsilon_{m n p q r} \partial^{r} \delta(1-2)} \\
& {\left[\triangleright_{m n}(1), \tilde{\triangleright}_{p q}(2)\right]=0} \\
& {\left[\tilde{\triangleright}_{m n}(1), \tilde{\triangleright}_{p q}(2)\right]=-2 i \epsilon_{m n p q r} \partial^{r} \delta(1-2) .}
\end{aligned}
$$

(Here "1" and "2" refer to points on the worldvolume, and $\delta(1-2)$ is shorthand for $\delta^{(5)}\left(\sigma_{1}-\right.$ $\left.\sigma_{2}\right)$.) Selfduality $(\tilde{\nabla}=0)$ is thus a second-class constraint (as for string scalars [28]), but we saw above the covariant division into first-class as $\frac{1}{4} \epsilon^{m n p q r} p_{n p} P_{q r}=\frac{1}{4} \epsilon^{m n p q r} p_{n p} \tilde{\triangleright}_{q r}$ (using a section condition). Unlike [27], the new constraint is linear in the string coordinates.

From the $\triangle \triangleright$ commutation relations we find

$$
\left[\mathcal{S}^{p}(1), \triangleright_{m n}(2)\right]=\frac{i}{2} \partial^{q} \delta(1-2) \delta_{[q}^{p} \triangleright_{m n]}(1) \quad \text { and } \quad\left[\mathcal{U}_{p}(1), \triangleright_{m n}(2)\right]=0 .
$$

Straighforward calculation gives

$$
\begin{aligned}
& {\left[\mathcal{S}^{m}(1), \mathcal{S}^{n}(2)\right]=i \partial^{(m} \delta(1-2) \mathcal{S}^{n)} \frac{1}{2}((1)+(2))-\frac{i}{2} \delta(1-2)\left[\partial^{[m} \mathcal{S}^{n]}-\epsilon^{m n p q r} \triangleright_{p q} \mathcal{U}_{r}\right]} \\
& {[\mathcal{S}, \mathcal{U}]=[\mathcal{U}, \mathcal{U}]=0}
\end{aligned}
$$

where we are defining $\mathcal{O} \frac{1}{2}((1)+(2)):=\frac{1}{2}[\mathcal{O}(1)+\mathcal{O}(2)]$.

On functions $f(X)=f(0)+\frac{1}{2} X^{m n} \partial_{m n} f(0)+\mathcal{O}\left(X^{2}\right)$, the Poisson brackets with the currents give spacetime derivatives

$$
\left[\triangleright_{m n}(1), f(X(2))\right]=-i \partial_{m n} f \delta(1-2)=\left[\tilde{\triangleright}_{m n}(1), f(X(2))\right] .
$$

The worldvolume version of this is given by

$$
\left[\left(\mathcal{S}^{m}-\tilde{\mathcal{S}}^{m}\right)(1), f(2)\right]=-i \partial^{m} f \delta(1-2)
$$

(with $\tilde{\mathcal{S}}$ formally the same as $\mathcal{S}$ but with $\triangleright$ replaced by $\tilde{\triangleright}$ ) so that, up to second-class constraints, $\mathcal{S}$ generates translations in $\sigma$. To see this, note that

$$
\partial^{r} f=\frac{1}{8} \epsilon^{m n p q r}\left(\partial_{m n} f\right)\left(\triangleright_{p q}-\tilde{\triangleright}_{p q}\right)-\left(\partial^{m} X^{n r}\right)\left(\partial_{m n} f\right)=\frac{1}{8} \epsilon^{m n p q r}\left(\partial_{m n} f\right)\left(\triangleright_{p q}-\tilde{\triangleright}_{p q}\right)
$$


modulo the new section condition (2.9c)

$$
\left(\partial^{n} f\right)\left(\partial_{m n} g\right)=0 \quad \forall f(X), g(X)
$$

(In T-theory the analogue of this formula for $\partial f$ was derived by dimensionally reducing (solving the second-class constraint) and then oxidizing [6].)

Worldvolume $(\sigma)$ reparameterizations are generated by

$$
\delta_{\xi}=i \int \xi_{m} \mathcal{S}^{m}
$$

Then (3.3) implies

$$
\delta_{\xi} \triangleright_{m n}=\frac{1}{2} \partial^{p}\left(\xi_{[p} \triangleright_{m n]}\right)=\left(\partial^{p} \xi_{p}\right) \triangleright_{m n}+\left(\partial^{p} \xi_{[m}\right) \triangleright_{n] p}+\xi_{p} \partial^{p} \triangleright_{m n}+\xi_{[m} \partial^{p} \triangleright_{n] p},
$$

corresponding to a density term (integrable on scalars), two contravariant index transformation terms, a coordinate transformation, and two terms for integrability on non-scalars. This demonstrates that $\triangleright$ isn't exactly a tensor, but its integral is invariant.

Using (3.7), we can compute the commutator of two vector fields $V_{i}^{m n}$ for ${ }_{i}={ }_{1,2}$ :

$$
\begin{aligned}
{\left[V_{1}^{m n} \triangleright_{m n}, V_{2}^{p q} \triangleright_{p q}\right] } & =2 i \epsilon_{m n p q r} \partial^{r} \delta(1-2) V_{1}^{m n} V_{2}^{p q} \frac{1}{2}((1)+(2)) \\
& -i \delta(1-2)\left[V_{[1}^{m n} \partial_{m n} V_{2]}^{p q}-\frac{1}{8} V_{[1}^{[m n} \partial_{m n} V_{2]}^{p q]}\right] \triangleright_{p q}
\end{aligned}
$$

modulo the second-class constraints (and sectioning). We use this to check the algebra of (spacetime) gauge transformations: defining

$$
\Lambda_{i}:=\frac{i}{2} \int \lambda_{i}^{m n} \triangleright_{m n}
$$

for $i_{i}{ }_{1}, 2$, we find for their commutator

$$
\left[\Lambda_{1}, \Lambda_{2}\right]=\frac{i}{2} \int\left[\frac{1}{2} \lambda_{[1}^{m n} \partial_{m n} \lambda_{2]}^{p q} \triangleright_{p q}-\frac{1}{16} \lambda_{[1}^{[m n} \partial_{m n} \lambda_{2]}^{p q]} \triangleright_{p q}\right]
$$

This shows that the algebra of gauge transformations (cf. ref. [9]) closes

$$
\begin{aligned}
{\left[\Lambda_{1}, \Lambda_{2}\right]=\Lambda_{12} \quad \text { with } \quad \lambda_{12}^{m n} } & =\frac{1}{2} \lambda_{[1}^{p q} \partial_{p q} \lambda_{2]}^{m n}-\frac{1}{16} \lambda_{[1}^{[m n} \partial_{p q} \lambda_{2]}^{p q]} \\
& =\frac{1}{4} \lambda_{[1}^{p q} \partial_{p q} \lambda_{2]}^{m n}-\frac{1}{2} \lambda_{[1}^{p[m} \partial_{p q} \lambda_{2]}^{n] q}-\frac{1}{4} \lambda_{[1}^{m n} \partial_{p q} \lambda_{2]}^{p q}
\end{aligned}
$$

Note that (3.7) implies that the gauge parameter itself has the gauge ambiguity

$$
\delta \lambda^{m n}=\frac{1}{2} \epsilon^{m n p q r} \partial_{p q} \lambda_{r} .
$$




\section{Backgrounds}

Background fields are introduced as usual through the covariant derivatives

$$
\triangleright_{a b}=\frac{1}{2} E_{a b}^{m n} \triangleright_{m n} .
$$

Then (3.11) applied to $\delta_{\lambda} \triangleright_{a b}=\left[\Lambda, \triangleright_{a b}\right]$ gives

$$
\begin{aligned}
\delta_{\lambda} E_{a b}{ }^{m n} & =\frac{1}{2} \lambda^{p q} \partial_{p q} E_{a b}{ }^{m n}-\frac{1}{2} E_{a b}{ }^{p q} \partial_{p q} \lambda^{m n}+\frac{1}{8} E_{a b}{ }^{[m n} \partial_{p q} \lambda^{p q]} \\
& =\frac{1}{2} \lambda^{p q} \partial_{p q} E_{a b}{ }^{m n}+\frac{1}{2} E_{a b}{ }^{m n} \partial_{p q} \lambda^{p q}+E_{a b}{ }^{p[m} \partial_{p q} \lambda^{n] q},
\end{aligned}
$$

in agreement with [9]. (There is also a nonderivative $\mathrm{Sp}(4)$ gauge transformation on the flat indices $a, b$.)

Factorization of the vielbein follows from requiring that the result $\mathcal{S}^{a}$ of replacing $\triangleright_{m n}$ with $\triangleright_{a b}$ in $\mathcal{S}$ does not generate an independent symmetry. This is essentially the statement that $\epsilon$ is a tensor under transformation by the vielbein, and thus the vielbein is an element of GL(5): introducing linear dependence through a new vielbein $E_{m}{ }^{a}$,

$$
\mathcal{S}^{a} \sim \mathcal{S}^{m} E_{m}{ }^{a} \Rightarrow \frac{1}{4} \epsilon^{a b c d e} E_{b c}{ }^{m n} E_{d e}{ }^{p q} \sim \epsilon^{m n p q r} E_{r}{ }^{a} \Rightarrow E_{a b}{ }^{m n}=E_{[a}{ }^{m} E_{b]}{ }^{n}
$$

where $E_{a}{ }^{m}$ is the inverse of $E_{m}{ }^{a}$, and we have chosen the proportionality factor to be $\operatorname{det}\left(E_{a}{ }^{m}\right)$ in the final step for convenience. Thus $E_{a}{ }^{m}$ and $E_{a b}{ }^{m n}$ are representations of GL(5) in the $\mathbf{5}$ and $\mathbf{1 0}$ representations, each of which has been expressed in terms of the other above.

Alternatively, using (3.11) again, one computes that

$$
\begin{aligned}
{\left[\triangleright_{a b}(1), \triangleright_{c d}(2)\right]=} & \frac{i}{2} \epsilon_{m n p q r} \partial^{r} \delta(1-2) E_{a b}{ }^{m n} E_{c d}{ }^{p q} \frac{1}{2}((1)+(2)) \\
& -\frac{i}{8} \delta(1-2)\left[E_{a b}{ }^{m n} \partial_{m n} E_{c d}{ }^{p q}-\frac{1}{8} E_{a b}{ }^{[m n} \partial_{m n} E_{c d}{ }^{p q]}-{ }_{a b} \leftrightarrow_{c d}\right] E_{p q}{ }^{e f} \triangleright_{e f} .
\end{aligned}
$$

Then the first term has to be proportional to $\epsilon_{a b c d e} E_{m}{ }^{e} \partial^{m} \delta(1-2)$. Since $E_{a}{ }^{m}$ is thus an unconstrained matrix, it's more convenient to use as the fundamental field. We therefore use (4.2) to find its gauge transformation:

$$
\delta E_{a}{ }^{m}=\frac{1}{2} \lambda^{p q} \partial_{p q} E_{a}{ }^{m}+\frac{1}{4} E_{a}{ }^{m} \partial_{p q} \lambda^{p q}+E_{a}{ }^{p} \partial_{p q} \lambda^{q m} .
$$

To rewrite (4.4) in terms of the fundamental field, it is useful to flatten the indices on the derivatives $\partial_{a b}:=E_{a}{ }^{m} E_{b}{ }^{n} \partial_{m n}$. The anholonomy coefficients

$$
\left[\partial_{a b}, \partial_{c d}\right]=\frac{1}{2}\left[c_{a b c d}^{e f}-c_{c d a b}^{e f}\right] \partial_{e f}
$$

reduce to $c_{a b c d}{ }^{e f}=\left(\partial_{a b} E_{[c}^{m}\right) E_{m}\left[e \delta_{d]}^{f]}=: c_{a b[c}\left[e \delta_{d]}^{f]}\right.\right.$. In terms of these, (4.4) becomes

$$
\begin{aligned}
{\left[\triangleright_{a b}(1), \triangleright_{c d}(2)\right]=} & \frac{i}{2} \delta(1-2)\left[c_{e[a b]}{ }^{e} \triangleright_{c d}+c_{a b[c}{ }^{e} \triangleright_{d] e}+c_{e[c \mid[a}{ }^{e} \triangleright_{b] \mid d]}+c_{[c \mid[a b]}{ }^{e} \triangleright_{\mid d] e}-([a b] \leftrightarrow[[c d])]\right. \\
& +2 i \operatorname{det}\left(E_{a}{ }^{m}\right) \epsilon_{a b c d e} \partial^{e} \delta(1-2),
\end{aligned}
$$

where $\partial^{a}:=E_{m}{ }^{a} \partial^{m}$ and we have rewritten $E_{a}{ }^{m} E_{b}{ }^{n} E_{c}{ }^{p} E_{d}^{q} E_{e}{ }^{r} \epsilon_{m n p q r}=\operatorname{det}\left(E_{a}{ }^{m}\right) \epsilon_{a b c d e}$. 


\section{$5 \quad$ Sections: $\mathrm{F} \rightarrow \mathrm{M}, \mathrm{T}, \mathrm{S}$}

Sectioning is straightforward. Solving the old section condition $\mathcal{S}_{0}^{m}$ as before, but also the new dimensional reduction condition $\stackrel{\circ}{\mathcal{S}^{m}}$, gives (for $m=-1,0,1,2,3$ )

$$
\begin{aligned}
& \epsilon^{m n p q r} p_{n p} p_{q r}=0 \quad \Rightarrow \quad p_{i j}=0 ; \quad p=p_{-1 i} \quad(i=0,1,2,3) \\
& \epsilon^{m n p q r} p_{n p} P_{q r}=0 \quad \Rightarrow \quad P_{i j}=0 ; \quad P=P_{-1 i} \\
& \triangleright_{-1 i}=P_{-1 i}, \quad \triangleright_{i j}=-\epsilon_{i j k l} \partial^{k} X^{-1 l} \\
& \mathcal{S}^{i}=\left(\partial^{[i} X^{-1 j]}\right) P_{-1 j}, \quad \mathcal{S}^{-1}=\frac{1}{2} \epsilon_{i j k l}\left(\partial^{i} X^{-1 j}\right)\left(\partial^{k} X^{-1 l}\right) \\
& \mathcal{U}_{i}=-\partial^{-1} P_{-1 i}, \quad \mathcal{U}_{-1}=\partial^{i} P_{-1 i}
\end{aligned}
$$

describing M-theory, still on a 5-brane, but in 4 spacetime dimensions.

On the other hand, solving the new conditions $\mathcal{U}$ and $\mathcal{U}_{0}$ gives

$$
\begin{aligned}
& \partial^{n} p_{m n}=0 \quad \Rightarrow \quad \partial^{i}=p_{3 i}=0 ; \quad \partial=\partial^{3}, \quad p=p_{i j} \quad(i=-1,0,1,2) \\
& \partial^{n} P_{m n}=0 \quad \Rightarrow \quad P_{3 i}=0 ; P=p_{i j} \\
& \triangleright_{3 i}=0, \quad \triangleright_{i j}=P_{i j}+\frac{1}{2} \epsilon_{i j k l} \partial^{3} X^{k l} \\
& \mathcal{S}^{i}=0, \quad \mathcal{S}^{3}=\frac{1}{8} \epsilon^{i j k l} \triangleright_{i j} \triangleright_{k l} \\
& \stackrel{\circ}{\mathcal{S}}^{i}=0, \quad \mathcal{S}^{3}=\frac{1}{4} \epsilon^{i j k l} p_{i j} P_{k l}
\end{aligned}
$$

describing T-theory on a 1-brane (string), in 6 (i.e., doubled) dimensions.

Solving both sets of conditions gives S-theory:

$$
\begin{aligned}
\partial=\partial^{3}, \quad p & =p_{-1 i} \quad(i=0,1,2) \\
P & =P_{-1 i} \\
\triangleright_{3 i}=0 ; \quad \triangleright_{-1 i} & =P_{-1 i}, \quad \triangleright_{i j}=-\epsilon_{i j k} \partial^{3} X^{-1 k} \\
\mathcal{S} & =\mathcal{S}^{3}=\left(\partial^{3} X^{-1 i}\right) P_{-1 i}
\end{aligned}
$$

\section{Conclusions}

Starting from the worldvolume theory of a selfdual gauge form in $\mathrm{D}=6$, we have derived the conditions (2.9) that generalize string theory to F-theory. All of them are new except for the section condition of F-gravity, which we have now found from first principles, along with the field representation of F-gravity. (Reduction to T-theory also gives a new covariant form for its dimensional reduction condition.) Their algebra follows from that of the (selfdual) currents (2.4), which generate the gauge transformations of F-gravity. (The constraints $\mathcal{S}^{m}$ generate $\sigma$ reparametrizations.) 
Many future avenues of investigation are suggested:

1. The covariant $6 \mathrm{D}$ conformal field theory might be useful, e.g., for analyzing $\alpha^{\prime}$ corrections. This would require an analysis of the algebra of the "T $\mathcal{T}$ " constraints in (2.7) responsible for the dynamics. A related problem is the 6D worldvolume vielbein: if part is identified with the spacetime vielbein, then the remainder might be the 6 gauge fields/Lagrange multipliers for worldvolume coordinate transformations.

2. Including the currents for $\mathrm{SO}(3,2)$ (or $\mathrm{SO}(3,3)$ for the $6 \mathrm{D}$ formulation) would allow the definition of truly covariant derivatives on the worldvolume and their torsion and curvature [34].

3. We have looked at only the bosonic string. Generalization to the superstring should be straightforward using [2]. Then reduction to T-theory can be compared to the formulation with Ramond-Ramond currents [35]. The tying of worldvolume and spacetime symmetries in the bosonic case suggests that the Green-Schwarz and Ramond-NeveuSchwarz formulations might be more directly related.

4. Of course, these results should be generalized to higher dimensions. However, simple use of selfdual forms would give different cosets than those found in the bosonic sectors of supergravities. Supersymmetry, especially for the $\mathrm{D}=4,6$, and 10 superstrings, should place new restrictions. For the $\mathrm{D}=4$ case, the bosonic coordinates are a spinor 16 representation of $\mathrm{SO}(5,5)$. Then the section condition uses a $10 \mathrm{D} \gamma$-matrix [36, 37]. The bosonic covariant derivative then resembles a fermionic supersymmetry-covariant derivative:

$$
\triangleright_{\mu}=P_{\mu}+\left(\gamma_{m}\right)_{\mu \nu} \partial^{m} X^{\nu} \Rightarrow\left[\triangleright_{\mu}, \triangleright_{\nu}\right]=2 i\left(\gamma_{m}\right)_{\mu \nu} \partial^{m} \delta
$$

Also,

$$
\mathcal{S}^{m}=\frac{1}{4}\left(\gamma^{m}\right)^{\mu \nu} \triangleright_{\mu} \triangleright_{\nu}
$$

\section{Acknowledgments}

WDL3 thanks Brenno Carlini Vallilo for discussions. WDL3 is partially supported by the UMcP Center for String \& Particle Theory and National Science Foundation grants PHY0652983, and PHY-0354401. Ws is supported in part by National Science Foundation grant PHY-1316617.

Open Access. This article is distributed under the terms of the Creative Commons Attribution License (CC-BY 4.0), which permits any use, distribution and reproduction in any medium, provided the original author(s) and source are credited.

\section{References}

[1] M. Poláček and W. Siegel, T-duality off shell in 3D Type II superspace, JHEP 06 (2014) 107 [arXiv: 1403.6904] [INSPIRE].

[2] W.D. Linch and W. Siegel, F-theory Superspace, arXiv:1501.02761 [INSPIRE]. 
[3] E. Witten, String theory dynamics in various dimensions, Nucl. Phys. B 443 (1995) 85 [hep-th/9503124] [INSPIRE].

[4] P. Hořava and E. Witten, Heterotic and type-I string dynamics from eleven-dimensions, Nucl. Phys. B 460 (1996) 506 [hep-th/9510209] [INSPIRE].

[5] W. Siegel, Two vierbein formalism for string inspired axionic gravity, Phys. Rev. D 47 (1993) 5453 [hep-th/9302036] [INSPIRE].

[6] W. Siegel, Superspace duality in low-energy superstrings, Phys. Rev. D 48 (1993) 2826 [hep-th/9305073] [INSPIRE].

[7] W. Siegel, Manifest duality in low-energy superstrings, in International Conference on Strings 93, pp. 353-363, 9, 1993 [hep-th/9308133] [INSPIRE].

[8] C. Vafa, Evidence for F-theory, Nucl. Phys. B 469 (1996) 403 [hep-th/9602022] [InSPIRE].

[9] D.S. Berman, H. Godazgar, M. Godazgar and M.J. Perry, The local symmetries of M-theory and their formulation in generalised geometry, JHEP 01 (2012) 012 [arXiv:1110.3930] [INSPIRE].

[10] D.S. Berman, H. Godazgar, M.J. Perry and P. West, Duality Invariant Actions and Generalised Geometry, JHEP 02 (2012) 108 [arXiv:1111.0459] [INSPIRE].

[11] D.S. Berman, H. Godazgar and M.J. Perry, $\mathrm{SO}(5,5)$ duality in M-theory and generalized geometry, Phys. Lett. B $\mathbf{7 0 0}$ (2011) 65 [arXiv:1103.5733] [InSPIRE].

[12] H. Godazgar, M. Godazgar, O. Hohm, H. Nicolai and H. Samtleben, Supersymmetric $E_{7(7)}$ Exceptional Field Theory, JHEP 09 (2014) 044 [arXiv: 1406.3235] [INSPIRE].

[13] E. Musaev and H. Samtleben, Fermions and supersymmetry in $E_{6(6)}$ exceptional field theory, JHEP 03 (2015) 027 [arXiv: 1412.7286] [INSPIRE].

[14] M. Hatsuda and K. Kamimura, M5 algebra and SO(5,5) duality, JHEP 06 (2013) 095 [arXiv: 1305.2258] [INSPIRE].

[15] M. Hatsuda and K. Kamimura, SL(5) duality from canonical M2-brane, JHEP 11 (2012) 001 [arXiv:1208.1232] [INSPIRE].

[16] M. Hatsuda and T. Kimura, Canonical approach to Courant brackets for D-branes, JHEP 06 (2012) 034 [arXiv: 1203.5499] [InSPIRE].

[17] J.-H. Park and Y. Suh, U-geometry: SL(5), JHEP 04 (2013) 147 [Erratum ibid. 11 (2013) 210] [arXiv: 1302.1652] [INSPIRE].

[18] C.D.A. Blair, E. Malek and J.-H. Park, M-theory and Type IIB from a Duality Manifest Action, JHEP 01 (2014) 172 [arXiv:1311.5109] [INSPIRE].

[19] C.D.A. Blair and E. Malek, Geometry and fluxes of SL(5) exceptional field theory, JHEP 03 (2015) 144 [arXiv:1412.0635] [INSPIRE].

[20] P.A.M. Dirac, An extensible model of the electron, Proc. Roy. Soc. Lond. A 268 (1962) 57 [inSPIRE].

[21] P.A. Collins and R.W. Tucker, Classical and Quantum Mechanics of Free Relativistic Membranes, Nucl. Phys. B 112 (1976) 150 [InSPIRE].

[22] P.S. Howe and R.W. Tucker, A Locally Supersymmetric and Reparametrization Invariant Action for a Spinning Membrane, J. Phys. A 10 (1977) L155 [inSPIRE].

[23] A. Sugamoto, Theory of Membranes, Nucl. Phys. B 215 (1983) 381 [InSPIRE]. 
[24] J. Hughes, J. Liu and J. Polchinski, Supermembranes, Phys. Lett. B 180 (1986) 370 [INSPIRE].

[25] E. Bergshoeff, E. Sezgin and P.K. Townsend, Supermembranes and Eleven-Dimensional Supergravity, Phys. Lett. B 189 (1987) 75 [INSPIRE].

[26] B. de Wit, J. Hoppe and H. Nicolai, On the Quantum Mechanics of Supermembranes, Nucl. Phys. B 305 (1988) 545 [INSPIRE].

[27] W. Siegel, Manifest Lorentz Invariance Sometimes Requires Nonlinearity, Nucl. Phys. B 238 (1984) 307 [INSPIRE].

[28] W. Siegel, Classical Superstring Mechanics, Nucl. Phys. B 263 (1986) 93 [INSPIRE].

[29] T. Kugo and B. Zwiebach, Target space duality as a symmetry of string field theory, Prog. Theor. Phys. 87 (1992) 801 [hep-th/9201040] [INSPIRE].

[30] W. Siegel, Randomizing the superstring, Phys. Rev. D 50 (1994) 2799 [hep-th/9403144] [INSPIRE].

[31] O. Hohm, W. Siegel and B. Zwiebach, Doubled $\alpha^{\prime}$-geometry, JHEP 02 (2014) 065 [arXiv: 1306.2970] [INSPIRE].

[32] A.A. Tseytlin, Duality Symmetric Formulation of String World Sheet Dynamics, Phys. Lett. B 242 (1990) 163 [inSPIRE].

[33] A.A. Tseytlin, Duality symmetric closed string theory and interacting chiral scalars, Nucl. Phys. B 350 (1991) 395 [INSPIRE].

[34] M. Poláček and W. Siegel, Natural curvature for manifest T-duality, JHEP 01 (2014) 026 [arXiv: 1308.6350] [INSPIRE].

[35] M. Hatsuda, K. Kamimura and W. Siegel, Ramond-Ramond gauge fields in superspace with manifest T-duality, JHEP 02 (2015) 134 [arXiv:1411.2206] [INSPIRE].

[36] A. Coimbra, C. Strickland-Constable and D. Waldram, $E_{d(d)} \times \mathbb{R}^{+}$generalised geometry, connections and M-theory, JHEP 02 (2014) 054 [arXiv:1112.3989] [INSPIRE].

[37] D.S. Berman, M. Cederwall, A. Kleinschmidt and D.C. Thompson, The gauge structure of generalised diffeomorphisms, JHEP 01 (2013) 064 [arXiv:1208.5884] [INSPIRE]. 